\title{
An optimal nuclei segmentation method based on enhanced multi-objective GWO
}

\author{
Ravi Sharma ${ }^{1} \cdot$ Kapil Sharma ${ }^{1}$
}

Received: 10 March 2021 / Accepted: 27 July 2021 / Published online: 4 October 2021

(c) The Author(s) 2021

\begin{abstract}
In breast cancer image analysis, reliable segmentation of the nuclei is still an open-ended research problem. In this paper, a new clustering-based nuclei segmentation method is presented. First, the proposed method pre-processes the histopathology image through SLIC method. Then, a novel variant of multi-objective grey wolf optimizer is employed to group the obtained super-pixels into optimal clusters. Lastly, the optimal cluster with minimum value is segmented as the nuclei region. The experimental results demonstrates that the proposed variant of multi-objective grey wolf algorithm surpasses the existing multiobjective algorithms over ten standard multi-objective benchmark functions belonging to different categories. Particularly, the proposed variant has achieved best fitness value of more than 0.90 on $90 \%$ of the considered functions. Further, the nuclei segmentation accuracy of the proposed method is validated on H\&E-stained estrogen receptor positive (ER+) breast cancer images. Experimental results illustrates that the proposed method has attained dice-coefficient value of more than 0.52 on $80 \%$ of the images. This illustrates that the proposed method is efficient in producing efficacious segmenting over histology images of Breast cancer.
\end{abstract}

Keywords Breast cancer diagnosis $\cdot$ Nuclei segmentation $\cdot$ Multi-objective grey wolf optimizer

\section{Introduction}

Histological images are the golden standard in the breast cancer diagnosis and hematoxylin and eosin (H\&E) staining of such images is the standard staining protocol [1]. In manual analysis of these images, there are a number of issues to be handled like, analysis variation due to difference in observer's experience, time-taking process, and difficult to identify subtle visual features [2]. However, the digitization of pathology systems have successfully mitigated such concerns [3]. In digital pathology, the segmentation of nuclei from the histopathological image is the foremost unit whose accuracy determines the efficiency of the system [4]. For the same, there are many nuclei segmentation methods defined over approaches like, super-pixels, clustering, active contours, watershed, and multi-level thresholding [5-7]. Among them, super-pixels is one of the efficient approaches for segmentation. Therefore, this paper introduces an efficient nuclei

\footnotetext{
Kapil Sharma

kapil@ieee.org

1 Delhi Technological University, Delhi, India
}

segmentation method based on super-pixels for histopathological images.

Super-pixels divide the image into non-overlapping regions wherein similar pixels are grouped together [8]. The boundary of each irregular-shaped super-pixel is according to the edge information in the original image. This makes each super-pixel perceptual meaningful $[9,10]$. Various computervision applications like, image segmentation [11], depth estimation [12], object localization [13], body model estimation [14], bag-of-features [15,16] and skeletonization [17], employ super-pixels to obtain mid-level representations. Fouad et al. [8] employed unsupervised learning on superpixels to segment a cancer image into different tissues. In literature, it has been observed that unsupervised learning is quite advantages for histopathological image analysis as these methods are efficient in identifying anatomical structures in an image [8,18].

Generally, unsupervised learning methods work on the principle of clustering the unlabeled data into homogeneous clusters according to the considered criteria such as intra-cluster distance [19-21]. Some of the popular unsupervised learning methods are KMeans and Fuzzy C-Means [22,23]. However, there are a number of demerits in such 


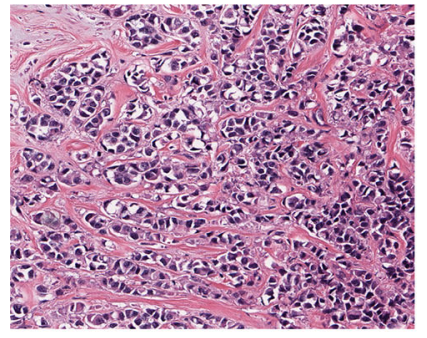

(a)

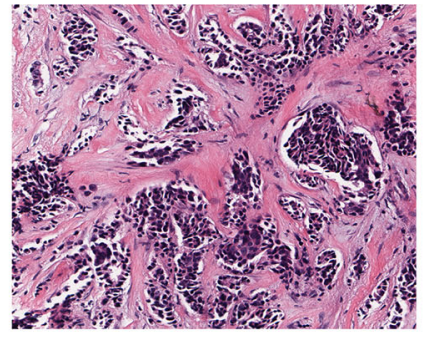

(b)

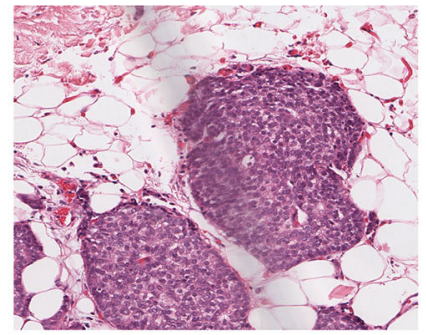

(c)

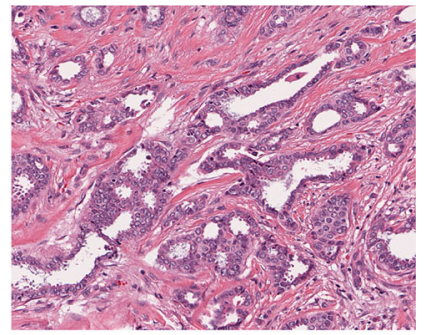

(d)

Fig. 1 Histopathological images scanned at $\times 40$ [47]

methods like, sensitive towards initial parameters settings, may return local optimal solutions, and require knowledge about cluster numbers to be formed [24-26]. To tackle these challenges, nature-inspired algorithms have proven a successful solution for generating efficient clusters [27,28]. Such algorithms have solved many optimization problems of real-world [29-32]. Some of the popular nature-inspired algorithms are genetic algorithm (GA) [33], biogeographybased optimization (BBO) [34,35], salp swarm optimization [36], gravitational search algorithm (GSA) [37], whale optimization algorithm (WOA) [38], and grey wolf optimizer (GWO) [39]. Moreover, it has been witnessed that natureinspired algorithms usually consider single objective like, intra-cluster distance, to perform clustering which presents only single view of the data $[40,41]$. To obtain better clusters, multi-objective criteria have been a better alternative as it presents multiple views while clustering the data which results in comparatively better segmented image. In literature, there are number of multi-objective natureinspired algorithms which try to optimizes multiple objective functions simultaneously. Some of popular multi-objective nature-inspired algorithms are Non-dominated sorting GA (NSGA-II) [42], Pareto-archived evolution strategy (PAES) [43], and strength-Pareto evolution algorithm (SPEA) [44]. Multi-objective GWO (MOGWO) is a recent multi-objective nature-inspired algorithm which has been inspired from the behavior of grey wolves. However, this algorithm suffers from a number of disadvantages like lacks population diversity, time consuming, and poor exploration capability. Therefore, Thus, the main contribution of this paper is fourfold; a novel variant of MOGWO, enhanced multi-objective grey wolf optimizer (EMOGWO), is proposed.

Further, this paper leverages the strengths of the proposed variant, EMOGWO, to segment the nuclei from histopathological images. However, nuclei segmentation in such images is a difficult task due to challenges like, non-uniformity in the nuclei shape, overlapping of nuclei, difference in tissue texture, different stain absorption capabilities of nucleus, and variation in scanned artifacts $[5,45,46]$. Figure 1 illustrates sample images, taken from a publicly available breast cancer histopathological dataset [47], to depict the involved complexity. In literature, multiple clustering criteria have been quite successful in handling such complex environment. Therefore, this paper present a new multi-objective clustering method, enhanced multi-objective grey wolf optimizer-superpixel (EMOGWO-SC), to cluster the superpixels optimally and efficiently to segment the nuclei from a histopathological image. Thus, the main contribution of this paper is fourfold:

- A new variant, enhanced multi-objective grey wolf optimizer (EMOGWO), is proposed.

- A novel multi-objective clustering method (EMOGWO$\mathrm{SC}$ ) is introduced which efficiently clusters the superpixels to segment the nuclei from a histopathological image.

- The proposed EMOGWO is compared against three other multi-objective nature-inspired algorithms on 10 well-known multi-objective benchmark. Thus, the main contribution of this paper is fourfold; problems in terms of IGD, SP and MS.

- To validate the performance of the proposed method (EMOGWO-SC), a publicly available dataset, H\&Estained estrogen receptor positive (ER+) breast cancer images $[47,48]$, has been considered and experimental comparison is conducted against GWO based superpixel clustering (GWO-SC) and kmeans based superpixel clustering (kmeans-SC) in terms of computation time and segmentation accuracy.

The remaining paper is organized as follows; "Preliminaries" briefs a super-pixel method and MOGWO. The proposed EMOGWO-SC along with EMOGWO is presented in "Proposed multi-objective clustering method for nuclei segmentation". Experimental analysis is conducted in "Results and discussion" followed by the conclusion in "Conclusion". 


\section{Preliminaries}

\section{SLIC: a super-pixel method}

Super-pixels are atomic and compact regions in an image that are formed after over-segmentation. Generally, superpixels are utilized for obtaining mid-level representation of an image [49]. One of the efficient method for generating superpixels is simple linear iterative clustering (SLIC) [10]. It demands only single input to operate, i.e., number of superpixels $(K)$ to be formed. In SLIC, two phases are followed, i.e., initialization and local clustering [10,50]. Initialization phase corresponds to the random initialization of $A$ centroids at an interval of $P=\sqrt{\frac{U}{A}}$. Here, $U$ represents the total pixels in an image. In local clustering phase, the distance $(T)$ of $k$ th super-pixel centroid from all the neighborhood pixels within the interval $2 P \times 2 P$ is measured according to Eq. (1).

$T(i, k)=\sqrt{\left(\frac{d_{\mathrm{c}}^{2}}{m}\right)+\left(\frac{d_{\mathrm{s}}^{2}}{S}\right)}$,

where $m$ corresponds to the constant and $i$ represents the $i$ th image pixel. For $k$ th super-pixel and $i$ th image pixel, $d_{\mathrm{c}}$ measures the Euclidean distance in the CIELab color space which is defined in Eq. (2), while $d_{\mathrm{s}}$ computes the Euclidean distance in the spatial space according to Eq. (3).

$d_{\mathrm{c}}(i, k)=\sqrt{\left(l_{i}-l_{k}\right)^{2}+\left(a_{i}-a_{k}\right)^{2}+\left(b_{i}-b_{k}\right)^{2}}$,

$d_{\mathrm{s}}(i, k)=\sqrt{\left(x_{i}-x_{k}\right)^{2}+\left(y_{i}-y_{k}\right)^{2}}$.

The measured value of $D$ is used to assign every pixel to the nearest super-pixel centroid. Then, mean of all the assigned pixels is computed to update the corresponding centroid of the super-pixels. This process is followed till residual error is converged. In post-processing, the unassigned pixels are assigned with nearest super-pixels.

\section{Multi-objective GWO}

Multi-objective grey wolf optimizer (MOGWO) [51] is the multi-objective version of grey wolf optimizer (GWO) [52]. The basic working of MOGWO is inspired from GWO only but there are two distinguishing two components in MOGWO. First component corresponds to the archive set and the other component is the leader selection. Pareto optimal solutions in an iteration are stored as archive set $(\mathrm{ACH})$ which depicts the set of solutions that are non-dominated by any other solution. Let an optimization problem $P$ need to optimize three objective functions, namely $f_{1}, f_{2}$, and $f_{3}$. Let there be two solutions, $X$ and $Y$. A solution $X$ dominates an another solution $Y$, if $X$ is better than $Y$ in at least one of the objectives $f_{i}$ and not worse than other objectives. In case, $X$ does not dominate $Y$ and $Y$ does not dominate $X$, they are called non-dominated solutions. These solutions are considered as better solutions than other solutions in the population.

In MOGWO, an archive controller is also maintained to control the movements of non-dominated solutions in archive set. Following rules of movement are followed on the archive set:

1. If the new solution $W$ is dominated by any solution of the $\mathrm{ACH}$, then $W$ can be added to $A R C$.

2. If the new solution $W$ dominates one or more solutions of the $\mathrm{ACH}$, then the dominated solution are deleted from the $\mathrm{ACH}$ and $W$ is added.

3. If both of the above conditions fail, then also $W$ is added.

4. If the $\mathrm{ACH}$ is full, the most crowded segments are identified using the grid mechanism and one of its solutions is deleted. Then, the solution $W$ is added to the least crowded segment to maintain the diversity.

To update the leader, leader selection method is applied on the updated ACH. Similar to GWO, the leaders, i.e., $\alpha, \beta$, and $\delta$ wolves are selected from the archive set which represent the three best solutions. For choosing the three best solution, fitness values are sorted and first three values are selected namely $\alpha, \beta$, and $\delta$. The other solutions in the population update the respective position according to these leaders only. Therefore, leader selection method is a key in the efficient performance of MOGWO.

For leader selection, a probability function $P_{i}$ is defined according to the density of the solution. Assume there are $S$ number of non-dominated solutions in $i$ th segment, then $P_{i}$ is defined as per Eq. (4).

$P_{i}=\frac{\text { const }}{S_{i}}$,

where const $>1$. From Eq. (4), it can be observed that the probability to pick a solution from highly crowded segments are lesser which is a good indication to maintain the diversity in the population [53]. This will redirect the search to less crowded segments and explore the search space for finding better solutions. Therefore, MOGWO maintains the diversity in the population and explores each region of the search space to find the optimal solutions. The position update equations and other steps of MOGWO algorithm according to GWO [52]. 


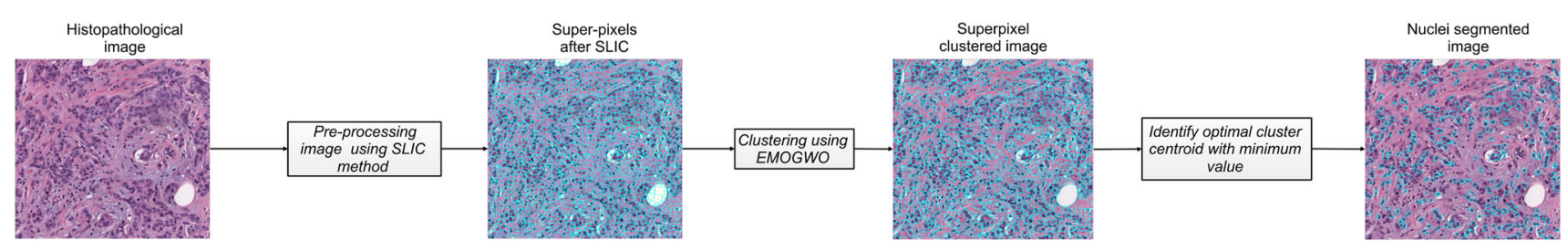

(a)

Fig. 2 Flow graph of the proposed multi-objective clustering method (IGSA-SC) for nuclei segmentation

\section{Proposed multi-objective clustering method for nuclei segmentation}

\section{Proposed method}

A new multi-objective clustering method, enhanced multiobjective grey wolf optimizer-based super-pixel clustering (EMOGWO-SC), is presented for optimal segmentation of nuclei from a histopathological image. The block diagram of the proposed method is illustrated in Fig. 2. First, a $\mathrm{H} \& \mathrm{E}$-stained histopathological image is pre-processed by the SLIC method to generate super-pixels. The generated super-pixels are further optimally clustered into ' $g$ ' clusters by employing the proposed enhanced multi-objective grey wolf optimizer (EMOGWO). For the same, two objective functions are considered, i.e., minimizing the intra-cluster distance and maximizing the inter-cluster distance. Intracluster distance measures the compactness of clusters while inter-cluster distance computes the separation among clusters. These two objective functions will help in achieving better clustering quality and optimal cluster centers. Therefore, the proposed method optimizes two objective functions simultaneously which are defined in Eqs. (5) and (6), respectively.

$$
\begin{aligned}
& \operatorname{Argmin}_{\left\{C_{1}, C_{2}, C_{3}, \ldots, C_{i}, \ldots, C_{g}\right\}}: \sum_{j=1}^{g} \sum_{i=1}^{p}\left\|C_{j}-x_{i}\right\|^{2}, \\
& \operatorname{Argmax}_{\left\{C_{1}, C_{2}, C_{3}, \ldots, C_{i}, \ldots, C_{g}\right\}}: \sum_{j=1}^{g} \sum_{i=1, i \neq j}^{g}\left\|C_{j}-C_{i}\right\|^{2},
\end{aligned}
$$

where ' $g$ ' and ' $p$ ' correspond to the number of required optimal clusters and total pixels in the image, respectively. Further, $C_{j}$ represent the $j$ th cluster centroid while $x_{i}$ is the $i$ th image pixel.

In a H\&E-stained histopathological image, nuclei regions are highlighted with dark color [54]. Therefore, the minimum average cluster is segmented as the nuclei region. The pseudo-code of the proposed method is presented in Algorithm 1. Further, the proposed variant, EMOGWO, is discussed in the following section.

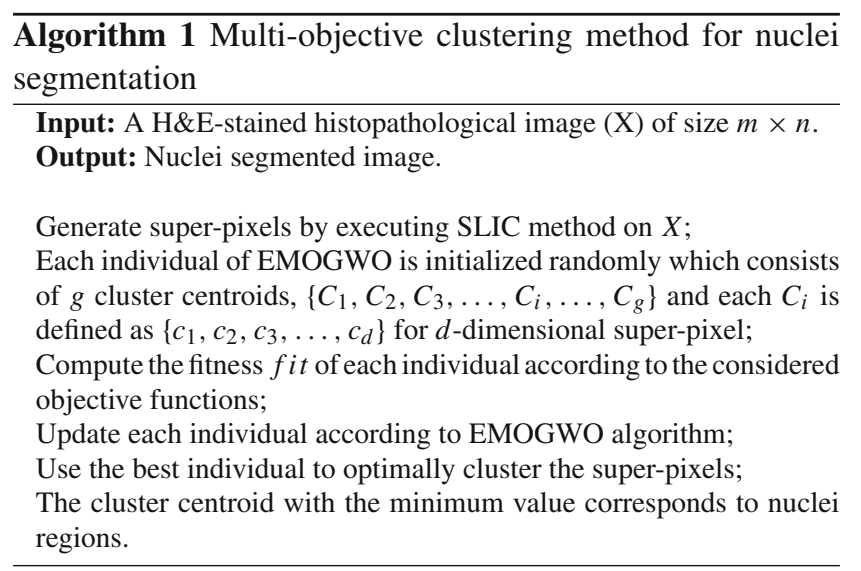

\section{Enhanced MOGWO (EMOGWO)}

In MOGWO, two new components have been introduced, namely archive set and leader selection method. The archive set contains the non-dominated solutions while leader selection method identifies three best solutions from least crowded segment in the population. However, this may lead to a problem. In case there are less than three solutions in the less crowded segment, then another least crowded segment will be evaluated to select the leaders. If this scenario remains same in the second less crowded segment also, then third less crowded segment will be selected. This results in increased time complexity. Therefore, this paper proposes an enhanced MOGWO (EMOGWO) with an enhanced leader selection method.

In the proposed method, the whole population is divided into different segments. The segment number is allocated to each solution $i$ based on Eq. (7).

$x_{i}=1+n_{i}$,

where $x_{i}$ represents segment number of solution $i$ and $n_{i}$ represents the number of solution that dominate solution $i$. Hence, for the non-dominated solutions the segment number will always be 1 . The maximum segment number will always be less than the total number solutions in the population. Once the population is divided into segments, less crowded solutions will be identified. Therefore, for every solution $i$ 
Table 1 Parameter settings for all the considered algorithms

\begin{tabular}{|c|c|c|c|c|c|}
\hline Sr. no. & Parameter & MOPSO & MOEA/D & MOGWO & EMOGWO \\
\hline 1. & Population size $(N)$ & 50 & 50 & 50 & 50 \\
\hline 2. & Number of iterations (itr) & 1000 & 1000 & 1000 & 1000 \\
\hline 3. & Number of search agents & 8 & 8 & 8 & 8 \\
\hline 4. & Number of repetitive runs & 10 & 10 & 10 & 10 \\
\hline 5. & Inertia weight $(w)$ & 0.8 & - & - & - \\
\hline 6. & Acceleration constants $(c 1, c 2)$ & 2 & - & - & - \\
\hline 7. & Grid inflation parameter $\left(\alpha_{p}\right)$ & 0.01 & - & - & - \\
\hline 8. & Leader selection pressure parameter $\beta_{p}$ parameter & 4 & - & - & - \\
\hline 9. & Mutation rate $(C R)$ & - & 0.5 & - & - \\
\hline 10. & Distribution index $(\eta)$ & - & 30 & - & - \\
\hline 11. & Probability of selecting parents $(\delta)$ & - & 0.9 & - & - \\
\hline 12. & $\alpha$ parameter & - & - & 0.99 & 0.99 \\
\hline 13. & $\beta$ parameter & - & - & 0.01 & 0.01 \\
\hline
\end{tabular}

in each segment, crowding count $\left(\mathrm{cc}_{i}\right)$ is calculated using Eq. (8)

$\mathrm{cc}_{i}=\sum_{j=1}^{\lambda\left(x_{i}\right)} h\left(d_{i j}\right)$

where $\lambda\left(x_{i}\right)$ is number of solutions in each segment number $x_{i}$ and $h\left(d_{i j}\right)$ is calculated by Eq. (9).

$h\left(d_{i j}\right)= \begin{cases}1-\left(\frac{d_{i j}}{\text { thres }}\right) & \text { if } d \leq \text { thres } \\ 0 & \text { Otherwise }\end{cases}$

where thres is selected between zero and one and may change based on the application while $d_{i j}$ is distance between two solutions $i$ and $j$ in the objective space having $M$ objectives. The calculation of distance is given by Eq. (10).

$d_{i j}=\sqrt{\sum_{o=1}^{M}\left(\frac{f_{o}^{i}-f_{o}^{j}}{f_{o}^{\max }-f_{o}^{\min }}\right)^{2}}$,

where $f_{o}^{\max }$ and $f_{o}^{\text {min }}$ are the maximum and minimum fitness value of $o$ th objective and $f_{o}^{i}, f_{o}^{j}$ are the fitness values of solutions $i$ and $j$ in $o$ th objective, respectively.

Now, the selection of three best solutions will be according to the roulette wheel selection, based on following probability for each segment.

$p_{i}^{k}=\frac{c}{\mathrm{cc}_{i}}$,

where $c$ is a constant greater than one and $\mathrm{cc}_{i}$ the crowding count of solution $i$ in the segment $k$.
From Eq. (11), it can be observed that if the $\mathrm{cc}_{i}$ is high, than the probability of this solution to be leader will be less. This indicates that less crowded solutions will be selected. This improves the population diversity and exploration capability of the algorithm.

\section{Results and discussion}

The performance of the proposed automatic nuclei segmentation method has experimented in two sections. First, "Performance analysis of EMOGWO" showcases the efficiency of the proposed multi-objective grey wolf optimizer on ten well-known CEC-2009 multi-objective benchmark functions [55] in which the proposed EMOGWO is validated qualitatively on 7 bi-objective and 3 tri-objective test problems. Second, in "Experimental analysis of automatic nuclei segmentation method", EMOGWO is used for nuclei segmentation within H\&E-stained breast cancer histology images. For a fair analysis, all the experimentation have been performed using Matlab 2017a on a system having $2.66 \mathrm{GHz}$ Intel core $\mathrm{i} 3$ processor and $8 \mathrm{~GB}$ of RAM.

\section{Performance analysis of EMOGWO}

The proposed EMOGWO has been tested over 10 multiobjective benchmark functions including 7 bi-objectives $\left(\mathrm{UF}_{1}-\mathrm{UF}_{7}\right)$ and 3 tri-objective test problems $\left(\mathrm{UF}_{8}-\mathrm{UF}_{10}\right)$ [55,56]. Tables 1 and 2 tabulate these benchmark functions along with definitions. The benchmark functions are considered as the most challenging test problems in the literature which include different multi-objective search region with non-convex, convex, multi-modal, and dis-continuous Pareto fronts. To assess the efficiency of the proposed EMOGWO, 
Table 2 Statistical results of all the considered algorithms for IGD on UF1-UF10

\begin{tabular}{|c|c|c|c|c|c|c|}
\hline Functions & Algorithms & Average & Median & STD. Dev. & Worst & Best \\
\hline \multirow[t]{4}{*}{ UF1 } & MOPSO & 0.1370 & 0.1317 & 0.0441 & 0.2279 & 0.0899 \\
\hline & MOEA/D & 0.1871 & 0.1829 & 0.0507 & 0.2464 & 0.1265 \\
\hline & MOGWO & 0.1144 & 0.1130 & 0.0195 & 0.1577 & 0.0802 \\
\hline & EMOGWO & 0.0858 & 0.0848 & 0.0147 & 0.1183 & 0.0602 \\
\hline \multirow[t]{4}{*}{ UF2 } & MOPSO & 0.0604 & 0.0484 & 0.0276 & 0.1305 & 0.0370 \\
\hline & MOEA/D & 0.1223 & 0.1201 & 0.0107 & 0.1437 & 0.1049 \\
\hline & MOGWO & 0.0583 & 0.0578 & 0.0074 & 0.0732 & 0.0498 \\
\hline & EMOGWO & 0.0437 & 0.0433 & 0.0055 & 0.0549 & 0.0374 \\
\hline \multirow[t]{4}{*}{ UF3 } & MOPSO & 0.3140 & 0.3080 & 0.0447 & 0.3777 & 0.2565 \\
\hline & MOEA/D & 0.2886 & 0.2893 & 0.0159 & 0.3029 & 0.2634 \\
\hline & MOGWO & 0.2557 & 0.2509 & 0.0807 & 0.3679 & 0.1295 \\
\hline & EMOGWO & 0.1918 & 0.1882 & 0.0605 & 0.3129 & 0.0971 \\
\hline \multirow[t]{4}{*}{ UF4 } & MOPSO & 0.1360 & 0.1343 & 0.0074 & 0.1519 & 0.1273 \\
\hline & MOEA/D & 0.0681 & 0.0685 & 0.0021 & 0.0704 & 0.0647 \\
\hline & MOGWO & 0.0587 & 0.0587 & 0.0005 & 0.0594 & 0.0580 \\
\hline & EMOGWO & 0.0440 & 0.0440 & 0.0004 & 0.0445 & 0.0435 \\
\hline \multirow[t]{4}{*}{ UF5 } & MOPSO & 2.2024 & 2.1257 & 0.5530 & 3.0384 & 1.4648 \\
\hline & MOEA/D & 1.2915 & 1.3376 & 0.1349 & 1.4675 & 1.1231 \\
\hline & MOGWO & 0.7971 & 0.6994 & 0.3786 & 1.7386 & 0.4680 \\
\hline & EMOGWO & 0.5978 & 0.5246 & 0.2839 & 1.3039 & 0.3510 \\
\hline \multirow[t]{4}{*}{ UF6 } & MOPSO & 0.3540 & 0.3873 & 0.2044 & 0.6151 & 0.0540 \\
\hline & MOEA/D & 0.4552 & 0.4377 & 0.1898 & 0.6770 & 0.0290 \\
\hline & MOGWO & 0.1604 & 0.0734 & 0.1391 & 0.4014 & 0.0628 \\
\hline & EMOGWO & 0.1674 & 0.0550 & 0.1043 & 0.3011 & 0.0471 \\
\hline \multirow[t]{4}{*}{ UF7 } & MOPSO & 0.3540 & 0.3873 & 0.2044 & 0.6151 & 0.0540 \\
\hline & MOEA/D & 0.4552 & 0.4377 & 0.1898 & 0.6770 & 0.0290 \\
\hline & MOGWO & 0.1604 & 0.0734 & 0.1391 & 0.4014 & 0.0628 \\
\hline & EMOGWO & 0.1427 & 0.0653 & 0.1238 & 0.3573 & 0.0558 \\
\hline \multirow[t]{4}{*}{ UF8 } & MOPSO & 0.5367 & 0.5364 & 0.1826 & 0.7964 & 0.2453 \\
\hline & MOEA/D & - & - & - & - & - \\
\hline & MOGWO & 2.0578 & 2.3360 & 1.1455 & 3.8789 & 0.4613 \\
\hline & EMOGWO & 0.5957 & 0.5954 & 0.2027 & 0.8840 & 0.2723 \\
\hline \multirow[t]{4}{*}{ UF9 } & MOPSO & 0.4885 & 0.4145 & 0.1445 & 0.7221 & 0.3336 \\
\hline & MOEA/D & - & - & - & - & - \\
\hline & MOGWO & 0.1917 & 0.1660 & 0.0925 & 0.4479 & 0.1291 \\
\hline & EMOGWO & 0.1630 & 0.1411 & 0.0786 & 0.3807 & 0.1097 \\
\hline \multirow[t]{4}{*}{ UF10 } & MOPSO & 3.5945 & 2.8255 & 3.4883 & 2.9564 & 1.0431 \\
\hline & MOEA/D & - & - & - & - & - \\
\hline & MOGWO & 1.6372 & 1.5916 & 0.2988 & 2.1622 & 1.2201 \\
\hline & EMOGWO & 1.4735 & 1.4325 & 0.2689 & 1.9460 & 1.0981 \\
\hline
\end{tabular}

inverted generational distance (IGD) that measures the convergence of all the considered algorithms has been used [57]. The maximum spread (MS) and spacing (SP) are used to compute and assess the coverage [44,58]. The mathematical equation of IGD is enhanced version of generational distance (GD) $[57,59]$ which is introduced by Sierra and Coello and formulated in Eq. (12).

$\mathrm{IGD}=\frac{\sqrt{\sum_{j=1}^{n} \mathrm{dis}_{j}^{2}}}{n}$,

where $n$ represents the true optimal Pareto solutions and $\operatorname{dis}_{j}$ refers to the Euclidean distance between the $j$ th true Pareto 
Table 3 Statistical results of all the considered algorithms for SP on UF1 to UF10

\begin{tabular}{|c|c|c|c|c|c|c|}
\hline \multirow[t]{4}{*}{ UF1 } & MOPSO & 0.0090 & 0.0086 & 0.0025 & 0.0146 & 0.0067 \\
\hline & MOEA/D & 0.0038 & 0.0038 & 0.0015 & 0.0067 & 0.0021 \\
\hline & MOGWO & 0.0124 & 0.0054 & 0.0146 & 0.0464 & 0.0008 \\
\hline & EMOGWO & 0.0128 & 0.0122 & 0.0035 & 0.0209 & 0.0096 \\
\hline \multirow[t]{4}{*}{ UF2 } & MOPSO & 0.0083 & 0.0081 & 0.0017 & 0.0125 & 0.0062 \\
\hline & MOEA/D & 0.0088 & 0.0086 & 0.0008 & 0.0104 & 0.0080 \\
\hline & MOGWO & 0.0096 & 0.0082 & 0.0031 & 0.0158 & 0.0066 \\
\hline & EMOGWO & 0.0111 & 0.0095 & 0.0036 & 0.0182 & 0.0076 \\
\hline \multirow[t]{4}{*}{ UF3 } & MOPSO & 0.0070 & 0.0068 & 0.0017 & 0.0101 & 0.0048 \\
\hline & MOEA/D & 0.0268 & 0.0251 & 0.0206 & 0.0626 & 0.0008 \\
\hline & MOGWO & 0.0459 & 0.0486 & 0.0145 & 0.0705 & 0.0155 \\
\hline & EMOGWO & 0.0519 & 0.0549 & 0.0164 & 0.0797 & 0.0175 \\
\hline \multirow[t]{4}{*}{ UF4 } & MOPSO & 0.0067 & 0.0066 & 0.0009 & 0.0081 & 0.0055 \\
\hline & MOEA/D & 0.0073 & 0.0073 & 0.0006 & 0.0084 & 0.0061 \\
\hline & MOGWO & 0.0097 & 0.0086 & 0.0039 & 0.0172 & 0.0058 \\
\hline & EMOGWO & 0.0109 & 0.0097 & 0.0044 & 0.0195 & 0.0066 \\
\hline \multirow[t]{4}{*}{ UF5 } & MOPSO & 0.0048 & 0.0049 & 0.0041 & 0.0121 & 0.0001 \\
\hline & MOEA/D & 0.0028 & 0.0001 & 0.0055 & 0.0162 & 0.0000 \\
\hline & MOGWO & 0.1523 & 0.0878 & 0.1625 & 0.5125 & 0.0084 \\
\hline & EMOGWO & 0.1706 & 0.0983 & 0.1820 & 0.5740 & 0.0094 \\
\hline \multirow[t]{4}{*}{ UF6 } & MOPSO & 0.0208 & 0.0124 & 0.0326 & 0.1114 & 0.0022 \\
\hline & MOEA/D & 0.0063 & 0.0000 & 0.0127 & 0.0303 & 0.0000 \\
\hline & MOGWO & 0.0145 & 0.0111 & 0.0125 & 0.0411 & 0.0019 \\
\hline & EMOGWO & 0.0165 & 0.0127 & 0.0142 & 0.0469 & 0.0022 \\
\hline \multirow[t]{4}{*}{ UF7 } & MOPSO & 0.0067 & 0.0066 & 0.0029 & 0.0124 & 0.0033 \\
\hline & MOEA/D & 0.0054 & 0.0044 & 0.0030 & 0.0117 & 0.0008 \\
\hline & MOGWO & 0.0082 & 0.0055 & 0.0086 & 0.0311 & 0.0003 \\
\hline & EMOGWO & 0.0093 & 0.0062 & 0.0097 & 0.0351 & 0.0004 \\
\hline \multirow[t]{4}{*}{ UF8 } & MOPSO & 0.0268 & 0.0264 & 0.0083 & 0.0447 & 0.0153 \\
\hline & MOEA/D & - & - & - & - & - \\
\hline & MOGWO & 0.0069 & 0.0047 & 0.0047 & 0.0188 & 0.0037 \\
\hline & EMOGWO & 0.0295 & 0.0290 & 0.0091 & 0.0492 & 0.0168 \\
\hline \multirow[t]{4}{*}{ UF9 } & MOPSO & 0.0234 & 0.0235 & 0.0041 & 0.0309 & 0.0172 \\
\hline & MOEA/D & - & - & - & - & - \\
\hline & MOGWO & 0.0174 & 0.0183 & 0.0063 & 0.0286 & 0.0065 \\
\hline & EMOGWO & 0.0195 & 0.0205 & 0.0071 & 0.0320 & 0.0073 \\
\hline \multirow[t]{4}{*}{ UF10 } & MOPSO & 0.0199 & 0.0207 & 0.0035 & 0.0267 & 0.0154 \\
\hline & MOEA/D & - & - & - & - & - \\
\hline & MOGWO & 0.0252 & 0.0239 & 0.0150 & 0.0538 & 0.0000 \\
\hline & EMOGWO & 0.0219 & 0.0227 & 0.0038 & 0.0293 & 0.0169 \\
\hline
\end{tabular}

optimal and the closest computed Pareto optimal solutions in the reference set.

The performance metric SP and MS can be mathematically formulated using Eqs. (13) and (14). where dis' is the mean of all dis ${ }_{j}, \mathrm{n}$ is the number of optimal Pareto solutions obtained so far, and $\operatorname{dis}_{j}=\min _{j}\left(\mid f_{1}^{i}(\mathbf{x})-\right.$ $\left.f_{1}^{j}(\mathbf{x})|+| f_{2}^{i}(\mathbf{x})-f_{2}^{j}(\mathbf{x}) \mid\right)$ for all $i, j=1,2,3, \ldots, n$.
$\mathrm{SP}=\sqrt{\frac{1}{n-1} \sum_{j=1}^{n}\left(\operatorname{dis}^{\prime}-\operatorname{dis}_{j}\right)^{2}}$,
$\mathrm{MS}=\sqrt{\sum_{j=1}^{t} \max \left(\operatorname{det}\left(a_{i}, b_{i}\right)\right)}$, 
Table 4 Statistical results of all the considered algorithms for MS on UF1-UF10

\begin{tabular}{|c|c|c|c|c|c|c|}
\hline Functions & Algorithms & Average & Median & STD. Dev. & Worst & Best \\
\hline \multirow[t]{4}{*}{ UF1 } & MOPSO & 0.6454 & 0.6632 & 0.1929 & 0.2659 & 0.9523 \\
\hline & MOEA/D & 0.5177 & 0.5954 & 0.1661 & 0.3149 & 0.7413 \\
\hline & MOGWO & 0.9268 & 0.9327 & 0.0688 & 0.8180 & 0.9971 \\
\hline & EMOGWO & 0.9361 & 0.9420 & 0.0695 & 0.8261 & 1.0000 \\
\hline \multirow[t]{4}{*}{ UF2 } & MOPSO & 0.9121 & 0.9164 & 0.0256 & 0.8665 & 0.9530 \\
\hline & MOEA/D & 0.8720 & 0.8744 & 0.0056 & 0.8599 & 0.8779 \\
\hline & MOGWO & 0.9097 & 0.9104 & 0.0287 & 0.8470 & 0.9479 \\
\hline & EMOGWO & 0.9734 & 0.9741 & 0.0307 & 0.9062 & 1.0000 \\
\hline \multirow[t]{4}{*}{ UF3 } & MOPSO & 0.6103 & 0.6161 & 0.1058 & 0.3817 & 0.7715 \\
\hline & MOEA/D & 0.2399 & 0.2294 & 0.1213 & 0.0898 & 0.4786 \\
\hline & MOGWO & 0.9498 & 1.0000 & 0.0878 & 0.7681 & 1.0000 \\
\hline & EMOGWO & 0.9783 & 1.0000 & 0.0904 & 0.7911 & 1.0000 \\
\hline \multirow[t]{4}{*}{ UF4 } & MOPSO & 0.8128 & 0.8132 & 0.0137 & 0.7944 & 0.8345 \\
\hline & MOEA/D & 0.8832 & 0.8813 & 0.0181 & 0.8532 & 0.9139 \\
\hline & MOGWO & 0.9424 & 0.9427 & 0.0009 & 0.9410 & 0.9433 \\
\hline & EMOGWO & 0.9613 & 0.9615 & 0.0009 & 0.9598 & 0.9621 \\
\hline \multirow[t]{4}{*}{ UF5 } & MOPSO & 0.2793 & 0.2865 & 0.0958 & 0.1557 & 0.4383 \\
\hline & MOEA/D & 0.2922 & 0.2917 & 0.0347 & 0.2383 & 0.3438 \\
\hline & MOGWO & 0.3950 & 0.4326 & 0.1749 & 0.0301 & 0.6104 \\
\hline & EMOGWO & 0.4622 & 0.5061 & 0.2047 & 0.0352 & 0.7142 \\
\hline \multirow[t]{4}{*}{ UF6 } & MOPSO & 0.2744 & 0.2292 & 0.1129 & 0.1544 & 0.5252 \\
\hline & MOEA/D & 0.0968 & 0.0001 & 0.2072 & 0.0000 & 0.5948 \\
\hline & MOGWO & 0.6736 & 0.7083 & 0.1232 & 0.3884 & 0.8149 \\
\hline & EMOGWO & 0.7881 & 0.8287 & 0.1442 & 0.4544 & 0.9535 \\
\hline \multirow[t]{4}{*}{ UF7 } & MOPSO & 0.4293 & 0.2952 & 0.2755 & 0.1446 & 0.8771 \\
\hline & MOEA/D & 0.5632 & 0.6327 & 0.2421 & 0.1496 & 0.9915 \\
\hline & MOGWO & 0.8013 & 0.9629 & 0.3087 & 0.0225 & 0.9875 \\
\hline & EMOGWO & 0.8814 & 1.0000 & 0.3395 & 0.0248 & 1.0000 \\
\hline \multirow[t]{4}{*}{ UF8 } & MOPSO & 0.5081 & 0.5060 & 0.1614 & 0.2272 & 0.7148 \\
\hline & MOEA/D & - & - & - & - & - \\
\hline & MOGWO & 0.4457 & 0.4443 & 0.1857 & 0.1886 & 0.8638 \\
\hline & EMOGWO & 0.4769 & 0.4754 & 0.1987 & 0.2018 & 0.9242 \\
\hline \multirow[t]{4}{*}{ UF9 } & MOPSO & 0.1982 & 0.1657 & 0.1635 & 0.0677 & 0.6424 \\
\hline & MOEA/D & - & - & - & - & - \\
\hline & MOGWO & 0.8399 & 0.9106 & 0.1976 & 0.2875 & 0.9375 \\
\hline & EMOGWO & 0.8819 & 0.9561 & 0.2075 & 0.3019 & 0.9844 \\
\hline \multirow[t]{4}{*}{ UF10 } & MOPSO & 0.1302 & 0.1091 & 0.0626 & 0.0649 & 0.2540 \\
\hline & MOEA/D & - & - & - & - & - \\
\hline & MOGWO & 0.2972 & 0.1424 & 0.3465 & 0.0319 & 0.9283 \\
\hline & EMOGWO & 0.3180 & 0.1523 & 0.3708 & 0.0342 & 0.9933 \\
\hline
\end{tabular}

where $\operatorname{det}()$ computes the Euclidean distance, $a_{j}, b_{j}$ represent the maximum and minimum value in $j$ th objective and $t$ is the total number of objectives.

The performance parameters SP, MS, and IGD quantitatively validate the efficacy as they compare the mean and standard deviation values of all the considered algorithms. Thus, to qualitatively validate the performance, the best set of Pareto optimal solutions of each algorithms is compared. To do comparative analysis, the proposed EMOGWO is compared with MOGWO [56], MOPSO [58], and MOEA/D [60]. To reduce the interference effect and for a fair analysis, each algorithm has been run 10 times. Moreover, the number of iterations (itr) and population size $(N)$ in all the considered algorithms are set as 1000 and 50, respectively, and 
Table 5 Mean ranking of all the considered methods

\begin{tabular}{lll}
\hline Rank & Methods & Rank value \\
\hline 1. & EMOGWO & $\mathbf{1 . 7 5}$ \\
2. & MOGWO & 2.08 \\
3. & MOEA/D & 2.73 \\
4. & MOPSO & 3.06 \\
\hline
\end{tabular}

all other parameters are taken from respective literature. To appraise the efficacy of the proposed EMOGWO, it is compared with all the considered algorithms in terms of mean, standard deviation, median, worst, and best values of IGD, MS, and SP. Tables 2, 3 and 4 depicts the IGD, SP, and MS values returned by the proposed EMOGWO and other considered algorithms. From the Table 2, it is observed that the proposed EMOGWO obtains the best IGD values for more than $90 \%$ of benchmark problems. As, IGD values are good indicators to benchmark the convergence of different algorithms. So, the results depicted in Table 2 signify the better convergence of the proposed EMOGWO. There are a few benchmark problems such as UF3, UF6, and UF7, in which MOEA/D obtains the best IGD values while MOPSO shows better results than proposed and other considered algorithms

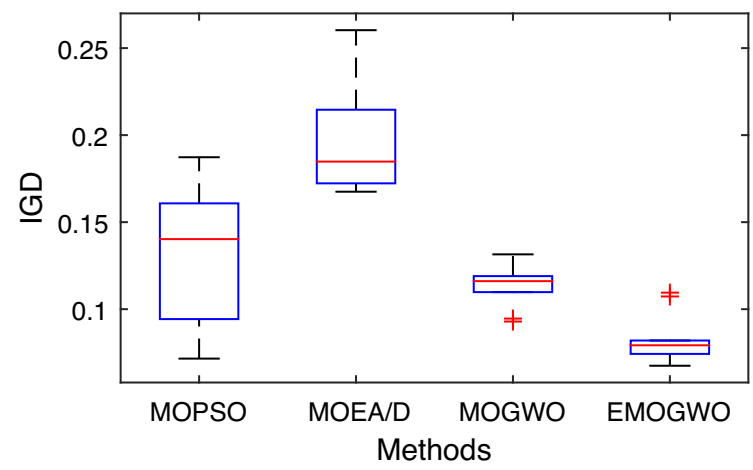

(a) for benchmark UF8. Thus, from the above analysis, it can be said that the performance of the proposed EMOGWO is more consistent than other considered algorithms.

Furthermore, SP and MS values are also compared in Tables 3 and 4. As MOEA/D is not implemented in Matlab for tri-objective benchmark problems UF8, Uf9, and UF10. Therefore, the efficiency of the proposed EMOGWO is only compared with MOPSO and MOGWO for these benchmark problems. It can be envisioned from the tables that the proposed EMOGWO show better coverage and convergence. Although, there are some discontinuities on the Pareto optimal front obtained by EMOGWO such as the coverage of whole front is broader than the MOGWO, MOPSO, and MOEA/D for most of the benchmark problems. However, the Pareto optimal solutions of the proposed EMOGWO are closer to the true Pareto optimal front and evenly distributed for both bi and tri-objectives. Thus, from the statistical results, efficacy of the proposed algorithm can be easily observed.

A Friedman's test [61] is also included in Table 5 to statistically validate the efficacy of the proposed EMOGWO. Friedman's test assesses the efficacy of each approach on each benchmark function and ranks them in order of effectiveness [62]. The best approach is given a score of 1, the

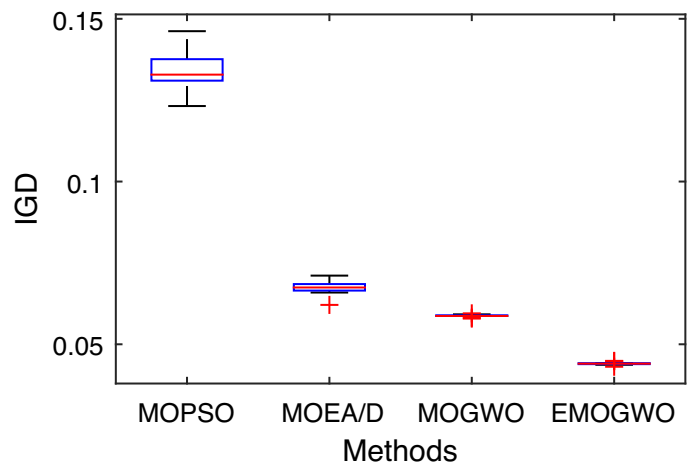

(b)

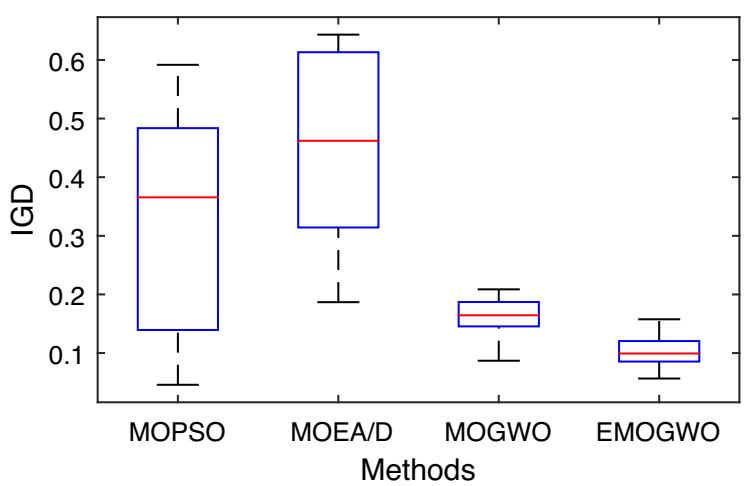

(c)

Fig. 3 Box-plots of the statistical results for IGD on three representative bi-modal benchmark problems a UF1, b UF4, and c UF6 


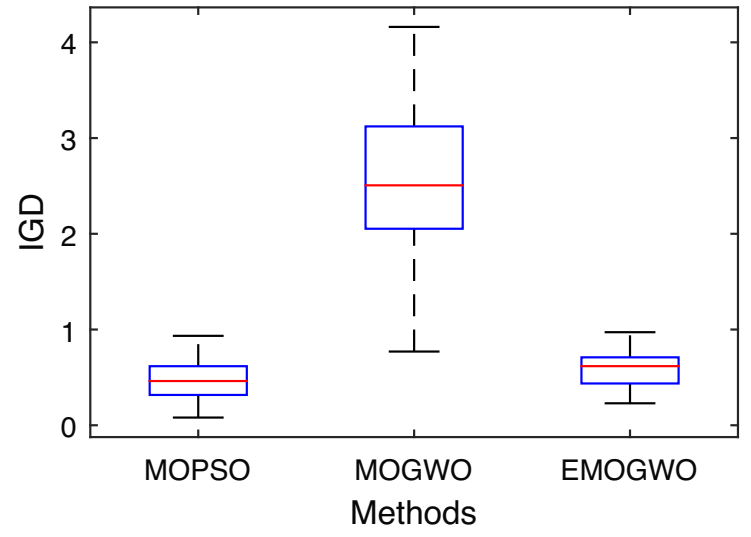

(a)

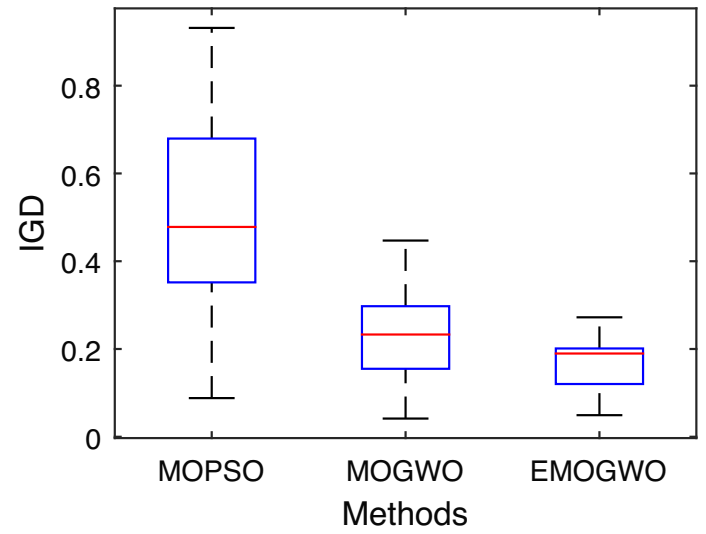

(b)

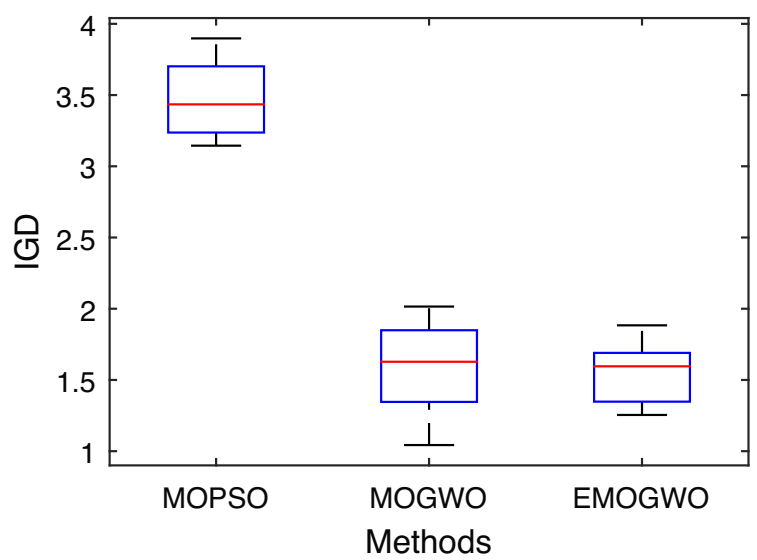

(c)

Fig. 4 Box-plots of the statistical results for IGD on three representative tri-modal benchmark problems a UF8, b UF9, and c UF10

second best is given a score of 2, the third best is given a score of 3 , and so on. The rank is calculated by averaging the ranks returned in different runs if the performance of the two approaches is identical [63]. Friedman test returns a $p$ value of 0.004756 , which is much lower than the threshold ( $\alpha=0.05$ ), indicating that the obtained results are statistically different. The ranks of all the methods returned by the Friedman test are tabulated in Table 5 which shows that the proposed EMOGWO has the lowest ranking value of all the models. As a consequence of the statistical analysis and experimental data, the EMOGWO is found to be superior.

Further, box-plots for 6 representative benchmark problems including 3 bi-modal (UF1, UF4, and UF6) and 3 tri-modal (UF8, UF9, and UF10) is also plotted in Figs. 3 and 4 to see the variations among the IGD values for existing and the proposed EMOGWO over 10 runs. In box-plots, algorithms are represented on the horizontal axis while the vertical axis denotes the best IGD values over 10 runs. It can be observed from the figures that the box-plots of the proposed EMOGWO in super narrow and its IGD value is also lower than MOPSO, POEA/D, and MOGWO for both bi-modal and tri-modal benchmark functions. However, for tri-modal benchmark function UF8, MOPSO shows comparative results. Hence, qualitative and quantitative analysis evident that the proposed EMOGWO is able to deliver very competing and promising results over multi-objective benchmark problems.

\section{Experimental analysis of automatic nuclei segmentation method}

In this section, the experimental results of the proposed multi-objective enhanced GWO-based automatic nuclei segmentation method (EMOGWO-SC) are presented. A breast cancer histology dataset which is publicly available [47] is considered for the performance evaluation. The dataset consists H\&E-stained ER+ (estrogen receptor positive) breast cancer images, taken at $40 \times$ magnifying level. The mask images are manual generated by the domain experts [47]. 


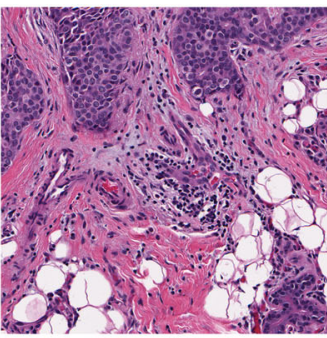

(a) Image1

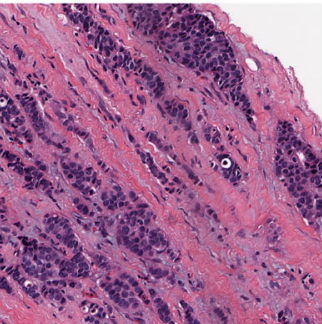

(f) Image2

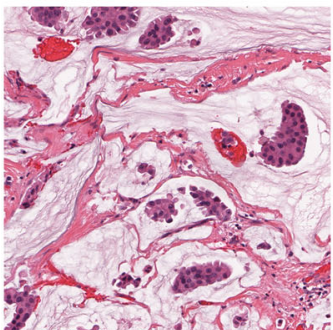

(k) Image3

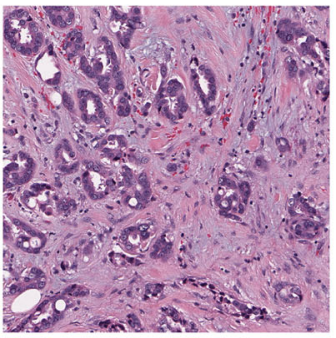

(p) Image4

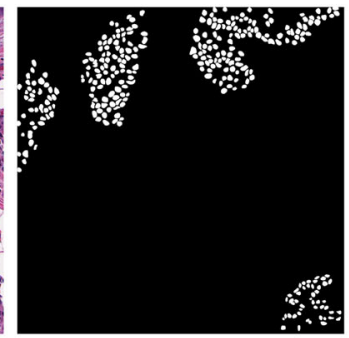

(b) Image 1: Mask

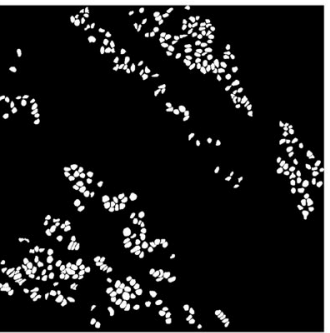

(g) Image 2: Mask

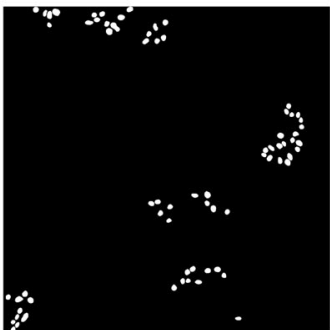

(l) Image3: Mask

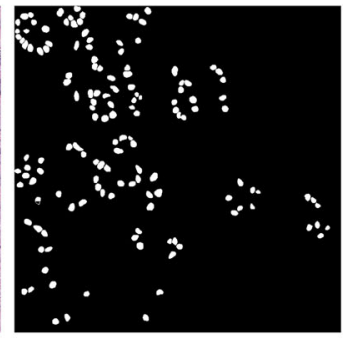

(q) Image4: Mask

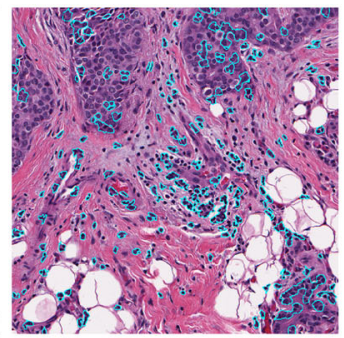

(c) Image1: EMOGWO$\mathrm{SC}$

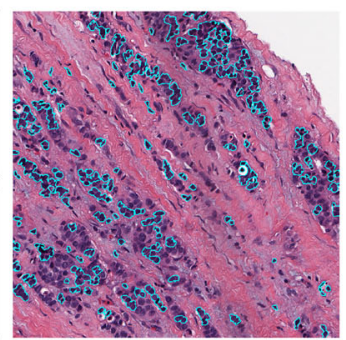

(h) Image2: EMOGWOSC

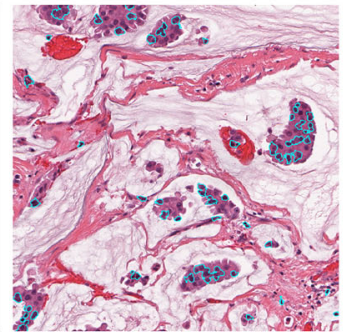

(m) EMOGWO-SC

Image3:

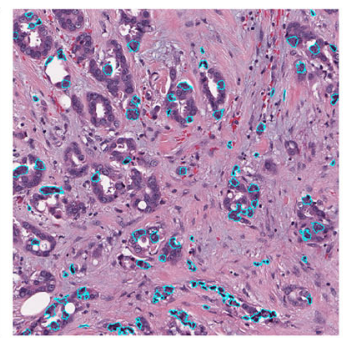

(r) Image4: EMOGWOSC
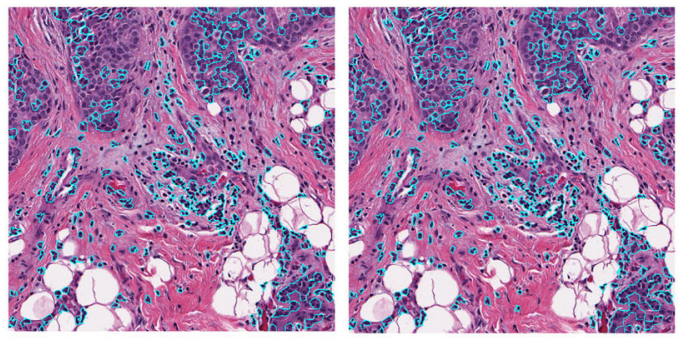

(d)
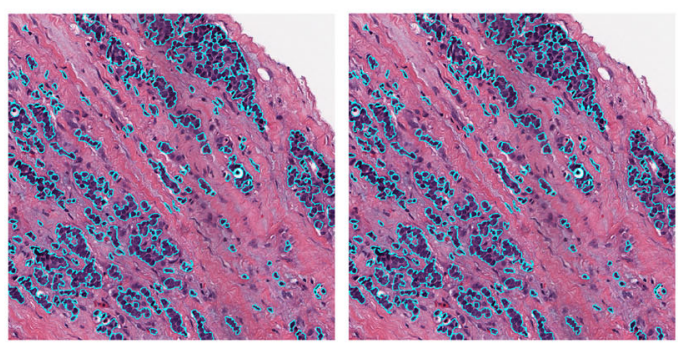

(j) Image2: kmeans-SC $\mathrm{SC}$
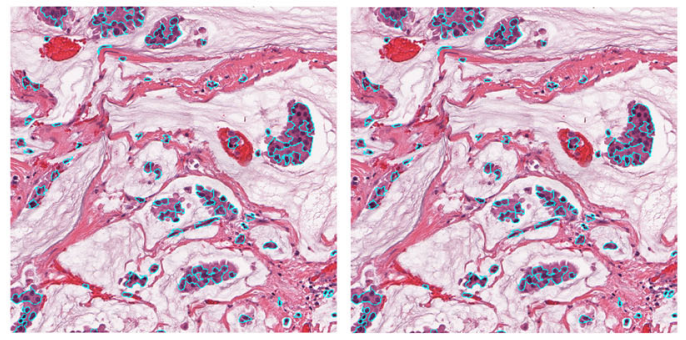

SC
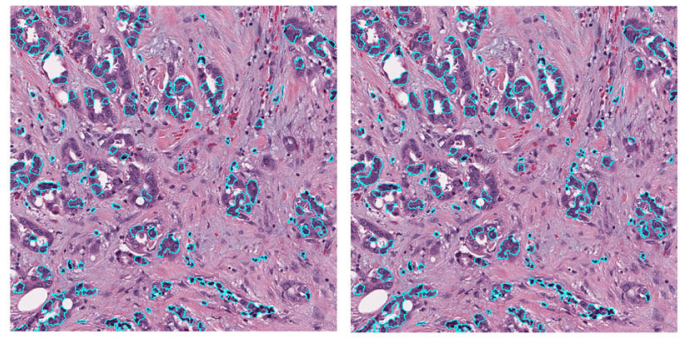

SC

(t) Image 4: kmeans-SC

Fig. 5 Nuclei segmentation results on representative H\&E-stained estrogen receptor positive (ER+) breast cancer images by the proposed and the considered methods

In Fig. 5, the first two columns illustrate sample images along with the corresponding mask. The images are reshaped to $64 \times 64$ resolution for the experimental purpose. The performance of the segmentation has been evaluated with four parameters, true positive (TP), true negative (TN), false negative (FN), and dice-coefficient. The experimental results are validated against two other state-of-the-art methods namely, K-means and GWO based superpixel method. The parameter setting of proposed and considered methods are taken from the respective literature.

The experimental results of proposed and considered methods for the segmented nuclei are performed on four randomly selected images as shown in Fig. 5. It is concluded from the results that the proposed EMOGWO based method performed superior for nuclei segmentation as compared to other considered methods. The higher numerical value of dice-coefficient represents better segmentation accu- 
Table 6 Segmentation accuracy results of the proposed and the considered methods on representative images

\begin{tabular}{|c|c|c|c|c|c|c|c|c|c|c|c|c|c|}
\hline \multirow[t]{2}{*}{ Images } & \multirow[t]{2}{*}{ Actual nuclei } & \multicolumn{4}{|c|}{ kmeans-SC } & \multicolumn{4}{|c|}{ MOGWO-SC } & \multicolumn{4}{|c|}{ EMOGWO-SC } \\
\hline & & $\overline{\mathrm{TP}}$ & FP & $\mathrm{FN}$ & $\mathrm{DC}$ & $\overline{\mathrm{TP}}$ & FP & FN & $\mathrm{DC}$ & $\overline{\mathrm{TP}}$ & FP & FN & $\overline{\mathrm{DC}}$ \\
\hline Image 1 & 33 & 32 & 56 & 3 & 0.4235 & 31 & 25 & 3 & 0.7420 & 32 & 13 & 1 & 0.6423 \\
\hline Image 2 & 35 & 30 & 36 & 4 & 0.3652 & 32 & 18 & 3 & 0.5820 & 34 & 6 & 1 & 0.5950 \\
\hline Image3 & 75 & 71 & 20 & 5 & 0.2463 & 72 & 14 & 4 & 0.5620 & 71 & 12 & 1 & 0.520 \\
\hline Image 4 & 29 & 22 & 49 & 4 & 0.3520 & 26 & 18 & 2 & 0.5216 & 27 & 16 & 1 & 0.5620 \\
\hline Image 5 & 28 & 24 & 7 & 3 & 0.3222 & 24 & 2 & 3 & 0.5020 & 23 & 3 & 2 & 0.5201 \\
\hline Image6 & 47 & 36 & 30 & 12 & 0.3960 & 35 & 28 & 13 & 0.5210 & 42 & 7 & 4 & 0.5306 \\
\hline Image 7 & 52 & 35 & 21 & 13 & 0.3256 & 37 & 16 & 14 & 0.5020 & 44 & 2 & 7 & 0.5120 \\
\hline Image 8 & 7 & 2 & 59 & 2 & 0.0895 & 2 & 3 & 2 & 0.4950 & 3 & 1 & 1 & 0.5560 \\
\hline Image9 & 77 & 55 & 24 & 18 & 0.4250 & 62 & 17 & 13 & 0.4920 & 64 & 5 & 11 & 0.4960 \\
\hline Image 10 & 59 & 41 & 75 & 19 & 0.3980 & 42 & 70 & 16 & 0.4560 & 45 & 37 & 15 & 0.4960 \\
\hline
\end{tabular}

promised in the nuclei segmentation applications. Also, the computation time of EMOGWO-SC is minimum among all the meta-heuristic-based methods.

\section{Conclusion}

In this paper, a new clustering-based nuclei segmentation method is introduced. The proposed method find the optimal cluster centroids using novel variant of multi-objective grey wolf optimizer. To perform optimal clustering, two objective functions, namely intra-cluster distance and inter-cluster distance, are considered. To validate the efficacy of the proposed variant, standard multi-objective functions have been considered which consists of 7 bi-objective and 3 tri-objective benchmark functions along with statistically investigation using box plots. It has been observed that the proposed variant is able to report best fitness value of more than 0.90 on $90 \%$ of the benchmark functions. Further, the proposed EMOGWO has been employed for nuclei segmentation from $\mathrm{H} \& \mathrm{E}$ stained estrogen receptor positive (ER+) breast cancer images. The results of the proposed method are empirically validated against $\mathrm{K}$-means-SC and MOGWO-SC in terms of segmentation accuracy. The experimental results demonstrate that EMOGWO-SC attained $D C$-value of more than 0.52 over $80 \%$ of the images and outperformed the considered methods on $90 \%$ of the images. Moreover, the average computation time of the proposed method is approximately 4 times less than the MOGWO-SC which is quite promising. In future, fuzzy based segmentation can also be inculcated. Also, the other criteria such as structural similarity, boundary displacement can be explored to tackle large datasets.

\section{Declarations}

Conflict of interest The authors have stated that this paper has no potential conflict of interest. 
Research involving human participants and/or animals This article does not contain any studies with human participants or animals performed by any of the authors.

\section{Informed consent N/A.}

Open Access This article is licensed under a Creative Commons Attribution 4.0 International License, which permits use, sharing, adaptation, distribution and reproduction in any medium or format, as long as you give appropriate credit to the original author(s) and the source, provide a link to the Creative Commons licence, and indicate if changes were made. The images or other third party material in this article are included in the article's Creative Commons licence, unless indicated otherwise in a credit line to the material. If material is not included in the article's Creative Commons licence and your intended use is not permitted by statutory regulation or exceeds the permitted use, you will need to obtain permission directly from the copyright holder. To view a copy of this licence, visit http://creativecomm ons.org/licenses/by/4.0/.

\section{References}

1. Pal R (2019) Enhancement of bag of features method for classification of histopathological images, Ph.D. dissertation, JIIT, Noida [Online]. https://shodhganga.inflibnet.ac.in/handle/10603/276586

2. Gurcan MN, Boucheron LE, Can A, Madabhushi A, Rajpoot NM, Yener B (2009) Histopathological image analysis: a review. IEEE Rev Biomed Eng 2:147-171

3. Louis DN, Feldman M, Carter AB, Dighe AS, Pfeifer JD, Bry L, Almeida JS, Saltz J, Braun J, Tomaszewski JE et al (2015) Computational pathology: a path ahead. Arch Pathol Lab Med 140:41-50

4. Mittal H, Saraswat M (2019) An automatic nuclei segmentation method using intelligent gravitational search algorithm based superpixel clustering. Swarm Evol Comput 45:15-32

5. Xing F, Yang L (2016) Robust nucleus/cell detection and segmentation in digital pathology and microscopy images: a comprehensive review. IEEE Rev Biomed Eng 9:234-263

6. Vink J, Van Leeuwen M, Van Deurzen C, De Haan G (2013) Efficient nucleus detector in histopathology images. J Microsc 249:124-135

7. Saraswat M, Arya K, Sharma H (2013) Leukocyte segmentation in tissue images using differential evolution algorithm. Swarm Evol Comput 11:46-54

8. Fouad S, Randell D, Galton A, Mehanna H, Landini G (2017) Unsupervised superpixel-based segmentation of histopathological images with consensus clustering. In: Lecture notes in annual conference on medical image understanding and analysis. Springer, Berlin, pp 767-779

9. Veksler O, Boykov Y, Mehrani P (2010) Superpixels and supervoxels in an energy optimization framework. In: Lecture notes in European conference on computer vision. Springer, Berlin, pp 211224

10. Achanta R, Shaji A, Smith K, Lucchi A, Fua P, Süsstrunk S (2012) SLIC superpixels compared to state-of-the-art superpixel methods. IEEE Trans Pattern Anal Mach Intell 34:2274-2282

11. He X, Zemel RS, Ray D (2006) Learning and incorporating topdown cues in image segmentation. In: Proceedings of European conference on computer vision. Springer, Berlin, pp 338-351

12. Hoiem D, Efros AA, Hebert M (2005) Automatic photo pop-up. ACM Trans Graph 24:577-584

13. Fulkerson B, Vedaldi A, Soatto S (2009) Class segmentation and object localization with superpixel neighborhoods. In: Proceedings of IEEE international conference on computer vision. IEEE, pp $670-677$

14. Mori G (2005) Guiding model search using segmentation. In: Proceedings of IEEE international conference on computer vision. IEEE, pp 1417-1423

15. Pal R, Saraswat M (2019) Grey relational analysis based keypoints selection in bag-of-features for histopathological image classification. Recent Pat Comput Sci 12(4):260-268

16. Pal R, Saraswat M (2019) Histopathological image classification using enhanced bag-of-feature with spiral biogeography-based optimization. Appl Intell 49(9):3406-3424

17. Levinshtein A, Sminchisescu C, Dickinson S (2013) Multiscale symmetric part detection and grouping. Int J Comput Vis 104:117134

18. Pal R, Saraswat M (2020) A new weighted two-dimensional vector quantisation encoding method in bag-of-features for histopathological image classification. Int J Intell Inf Database Syst 13(24):150-171

19. Gupta R, Pal R (2018) Biogeography-based optimization with lévyflight exploration for combinatorial optimization. In: International conference on cloud computing, data science \& engineering (Confluence), pp 664-669

20. Himabindu G, Murty MR et al (2018) Extraction of texture features and classification of renal masses from kidney images. Int J Eng Technol 7(2):1057-1063

21. Pal R, Sharma AK (2014) MSEP-E: enhanced stable election protocol with multihop communication. Glob J Comput Sci Technol $14: 1-8$

22. Mittal H, Saraswat M (2020) A new fuzzy cluster validity index for hyper-ellipsoid or hyper-spherical shape close clusters with distant centroids. IEEE Trans Fuzzy Syst. https://doi.org/10.1109/ TFUZZ.2020.3016339

23. Bhushan S, Pal R, Antoshchuk SG (2018) Energy efficient clustering protocol for heterogeneous wireless sensor network: a hybrid approach using GA and K-means. In: 2018 IEEE second international conference on data stream mining and processing (DSMP). IEEE, pp 381-385

24. Mittal H, Pandey AC, Pal R, Tripathi A (2021) A new clustering method for the diagnosis of CoVID19 using medical images. Appl Intell 51(5):2988-3011

25. Pal R, Saraswat M (2017) Data clustering using enhanced biogeography-based optimization. In: 2017 Tenth international conference on contemporary computing (IC3). IEEE, pp 1-6

26. Himabindu G, Ramakrishna Murty M et al (2018) Classification of kidney lesions using bee swarm optimization. Int J Eng Technol 7(2.33):1046-1052

27. Pandey AC, Pal R, Kulhari A (2018) Unsupervised data classification using improved biogeography based optimization. Int J Syst Assur Eng Manag 9(4):821-829

28. Mittal H, Saraswat M (2018) An image segmentation method using logarithmic kbest gravitational search algorithm based superpixel clustering. Evol Intel. https://doi.org/10.1007/s12065-018-0192

29. Mittal H, Saraswat M, Bansal JC, Nagar A, Fake-face image classification using improved quantum-inspired evolutionary-based feature selection method. In: 2020 IEEE symposium series on computational intelligence (SSCI). IEEE, pp 989-995 (2020)

30. Mittal H, Saraswat M, Pal R (2020) Histopathological image classification by optimized neural network using IGSA. In: International conference on distributed computing and internet technology. Springer, Berlin, pp 429-436

31. Mittal H, Saraswat M (2019) Classification of histopathological images through bag-of-visual-words and gravitational search algorithm. In: Soft computing for problem solving. Springer, Berlin, pp 231-241

32. Sri MN, Priyanka JH, Sailaja D, Murthy MR (2019) A comparative analysis of breast cancer data set using different classification meth-

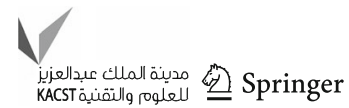


ods. In: Smart intelligent computing and applications. Springer, Berlin, pp 175-181

33. Raju P, Subash Y, Rishabh K (2020) EEWC: energy-efficient weighted clustering method based on genetic algorithm for HWSNs. Complex Intell Syst 6(2):391-400

34. Pal R, Mittal H, Saraswat M (2019) Optimal fuzzy clustering by improved biogeography-based optimization for leukocytes segmentation. In: 2019 Fifth international conference on image information processing (ICIIP). IEEE, pp 74-79

35. Pal R, Saraswat M (2018) Enhanced bag of features using AlexNet and improved biogeography-based optimization for histopathological image analysis. In: 2018 Eleventh international conference on contemporary computing (IC3). IEEE, pp 1-6

36. Pandey AC, Tripathi AK, Pal R, Mittal H, Saraswat M (2019) Spiral salp swarm optimization algorithm. In: 4th International conference on information systems and computer networks, pp 722-727

37. Mittal H, Tripathi A, Pandey AC, Pal R (2021) Gravitational search algorithm: a comprehensive analysis of recent variants. Multimedia Tools Appl 80(5):7581-7608

38. Tripathi AK, Mittal H, Saxena P, Gupta S (2021) A new recommendation system using map-reduce-based tournament empowered Whale optimization algorithm. Complex Intell Syst 7(1):297-309

39. Jaiswal K, Mittal H, Kukreja S (2017) Randomized grey wolf optimizer (RGWO) with randomly weighted coefficients. In: 2017 Tenth international conference on contemporary computing (IC3). IEEE, pp 1-3

40. Mittal H, Saraswat M (2018) cKGSA based fuzzy clustering method for image segmentation of RGB-D images. In: 2018 Eleventh international conference on contemporary computing (IC3). IEEE, pp 1-6

41. Pal R, Saraswat M (2018) A new bag-of-features method using biogeography-based optimization for categorization of histology images. Int J Inf Syst Manag Sci 1(2)

42. Deb K, Pratap A, Agarwal S, Meyarivan T (2002) A fast and elitist multiobjective genetic algorithm: NSGA-II. IEEE Trans Evol Comput 6(2):182-197

43. Knowles J, Corne D (1999) The Pareto archived evolution strategy: a new baseline algorithm for Pareto multiobjective optimisation. In: Proceedings of the 1999 Congress on evolutionary computationCEC99 (Cat. No. 99TH8406), vol 1. IEEE, pp 98-105

44. Zitzler E, Thiele L (1999) Multiobjective evolutionary algorithms: a comparative case study and the strength Pareto approach. IEEE Trans Evol Comput 3(4):257-271

45. Veta M, Pluim JP, Van Diest PJ, Viergever MA (2014) Breast cancer histopathology image analysis: a review. IEEE Trans Biomed Eng 61:1400-1411

46. Veta M, Huisman A, Viergever MA, van Diest PJ, Pluim JP (2011) Marker-controlled watershed segmentation of nuclei in H\&E stained breast cancer biopsy images. In: Proceedings of IEEE biomedical imaging: from nano to macro. IEEE, pp 618-621

47. Use case 1: Nuclei segmentation-Andrew Janowczyk. http:// www.andrewjanowczyk.com/use-case-1-nuclei-segmentation/. Accessed 20 Dec 2020

48. Janowczyk A, Madabhushi A (2016) Deep learning for digital pathology image analysis: a comprehensive tutorial with selected use cases. J Pathol Inform 7:17-42

49. Arisoy S, Kayabol K (2016) Mixture-based superpixel segmentation and classification of SAR images. IEEE Geosci Remote Sens Lett 13:1721-1725

50. Bao J, Yin J, Yang J (2017) Superpixel-based segmentation for multi-temporal PolSAR images. In: Proceedings of IEEE progress in electromagnetics research symposium-fall. IEEE, pp 654-658
51. Mirjalili S, Saremi S, Mirjalili SM, dos L, Coelho S (2016) Multiobjective grey wolf optimizer: a novel algorithm for multi-criterion optimization. Expert Syst Appl 47:106-119

52. Mirjalili S, Lewis A (2014) Adaptive gbest-guided gravitational search algorithm. Neural Comput Appl 25:1569-1584

53. Kulhari A, Pandey A, Pal R, Mittal H (2016) Unsupervised data classification using modified cuckoo search method. In: 2016 Ninth international conference on contemporary computing (IC3). IEEE, pp $1-5$

54. Yi F, Huang J, Yang L, Xie Y, Xiao G (2017) Automatic extraction of cell nuclei from H\&E-stained histopathological images. J Med Imaging 4:27-30

55. Zhang Q, Zhou A, Zhao S, Suganthan PN, Liu W, Tiwari S (2008) Multiobjective optimization test instances for the CEC 2009 special session and competition

56. Mirjalili S, Saremi S, Mirjalili SM, Coelho LdS (2016) Multiobjective grey wolf optimizer: a novel algorithm for multi-criterion optimization. Expert Syst Appl 47:106-119

57. Van Veldhuizen DA, Lamont GB (1998) Multiobjective evolutionary algorithm research: a history and analysis. Technical Report, Citeseer

58. Coello CAC, Pulido GT, Lechuga MS (2004) Handling multiple objectives with particle swarm optimization. IEEE Trans Evol Comput 8(3):256-279

59. Fonseca CM, Knowles JD, Thiele L, Zitzler E (2005) A tutorial on the performance assessment of stochastic multiobjective optimizers. In: Third international conference on evolutionary multi-criterion optimization (EMO 2005), vol 216, p 240

60. Zhang Q, Li H (2007) MOEA/D: a multiobjective evolutionary algorithm based on decomposition. IEEE Trans Evol Comput 11(6):712-731

61. Zimmerman DW, Zumbo BD (1993) Relative power of the Wilcoxon test, the Friedman test, and repeated-measures ANOVA on ranks. J Exp Educ 62(1):75-86

62. Xu J, Shan G, Amei A, Zhao J, Young D, Clark S (2017) A modified Friedman test for randomized complete block designs. Commun Stat Simul Comput 46(2):1508-1519

63. Sepanski JH (2007) A modification on the Friedman test statistic. Commun Stat Simul Comput ${ }^{\circledR}$ 36(4):783-790

Publisher's Note Springer Nature remains neutral with regard to jurisdictional claims in published maps and institutional affiliations. 